\title{
NEW SIMULATION RESULTS FOR THE S-DALINAC ELECTRON SOURCE*
}

\author{
U. Becker, $\underline{\text { P. Schütt }}^{\dagger}$, S. Setzer, A. Skocic, T. Weiland, \\ Theorie elektromagnetischer Felder, TU-Darmstadt \\ R. Eichhorn, H.-D. Gräf, S. Kostial, A. Richter, Institut für Kernphysik, TU-Darmstadt
}

\section{Abstract}

For the operation of its mid-infrared FEL the superconducting electron accelerator S-DALINAC has to produce bunches with a charge of $6 \mathrm{pC}$ and a duration of $2 \mathrm{ps}$ FWHM. In order to achieve these figures a subharmonic injection scheme was added to the $3 \mathrm{GHz} \mathrm{cw}$ accelerator. The electron gun consists of a grid controlled thermionic cathode on a $250 \mathrm{kV}$ terminal. It produces electron pulses with a charge of $20 \mathrm{pC}$ and a width of 800 ps FWHM at a repetition rate of $10 \mathrm{MHz}$. For a reliable simulation of the subsequent parts of the injector $(600 \mathrm{MHz}$ chopper/prebuncher section and a superconducting linac consisting of 2- and 5-cell capture cavities and two 20-cell accelerating structures) the beam parameters at its input have to be known as exactly as possible.

Since we expect that due to the pulsed operation of the gun, longitudinal space charge effects might be important, the electron gun and the $250 \mathrm{kV}$ electrostatic acceleration have been studied using the $21 / 2$ dimensional PIC code MAFIA-TS2, and the tracking code GPT.

This calculation takes into account the time structure of the beam and yields results like bunch shape at the gun exit, correlated and uncorrelated energy spread, as well as the transverse emittance.

The results of the simulation as well as a comparison with measurements of the transverse emittance and the electron pulse length at the exit of the electrostatic acceleration will be presented in this paper.

\section{MOTIVATION}

The generation of an electron beam with a peak current of 2.7 A for the operation of the FEL is a big challenge for the S-DALINAC, originally designed for a cw beam with low bunch charge. The necessary bunching in the injector was calculated before[1] using beam parameters at the exit of the gun resulting from a time independent simulation (DC emission). While transverse beam parameters were determined earlier, the longitudinal phase space was not known.

However, these longitudinal parameters are essential for the achievable peak current and therefore for the laser operation. The simulation results presented in this paper, which

\footnotetext{
* Supported by DFG under Contract Nos. Ri 242/12-1, SCHU 1241/11 and through the Graduiertenkolleg "Physik und Technik von Beschleunigern".

† Email: schuett@temf.tu-darmstadt.de
}

include energy spread and bunch length, are crucial for understanding particle dynamics in the buncher section and injector linac. Therefore extensive tracking calculations for these sections already have been started.

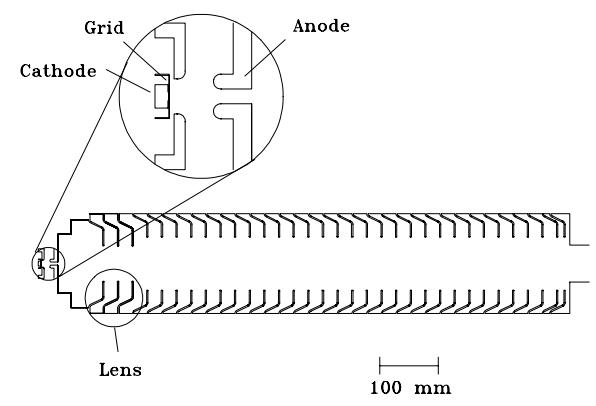

Figure 1: The S-DALINAC electron source.

The layout of the electron gun is shown in Fig. 1. The time structure of the emission from the thermionic gun is controlled by a grid in a distance of $150 \mu \mathrm{m}$. The pulse length is $\sigma_{t}=300 \mathrm{ps}$. Downstream of the anode, the beam is focused by an electrostatic lens and then accelerated to $250 \mathrm{keV}$ in an $80 \mathrm{~cm}$ long electrostatic cascade.

\section{SIMULATION METHODS}

For the simulation of this electron gun, it is necessary to include space-charge forces. Since we are interested in longitudinal phase space, we must choose a program, that works in time domain. Two different computer codes were used for the calculation:

\subsection{The PIC Codes MAFIA-TS2 and-TS3}

TS2 and TS3 are the names of the Particle in Cell (PIC) modules in MAFIA[2]. They have been used successfully for the computer aided design of klystrons and other RF sources[3]. The PIC programs solve Maxwell's equations in the time domain with a FDTD algorithm and in parallel, selfconsistently, integrate the equations of motion of the charged particles. These particles, in turn, are the source of the electromagnetic fields.

TS2 is the axisymmetric, two dimensional version, TS3 works with three dimensional cartesian coordinates. Both are fully relativistic and due to their basic physics algorithm a priori include all interaction of the charged particles and electromagnetic fields. Therefore, space charge, self magnetic fields, wake fields etc. are all included. Furthermore, 
both codes can use precalculated fields, which are provided by other solvers in the MAFIA package.

However, these PIC codes are very expensive to use. Especially, for very low particle energies close to the cathode, a fine resolution of the calculation grid is necessary and many particles must be used. Therefore, we also used a faster tracking code and compared the results.

\subsection{The Tracking Code GPT}

The General Particle Tracer (GPT)[4] code is a well established 3D tracking program. It includes the calculation of space-charge forces in a point-to-point model. Its integration method is based on a fifth order embedded RungeKutta method with adaptive step size control.

The field pattern in the gun was calculated in MAFIA-S and read into GPT via our own interface.

\section{RESULTS OF THE SIMULATION}

\subsection{Initial Conditions}

The thermionic cathode is operated at a temperature of $1100^{\circ} \mathrm{C}$. Therefore the mean energy of the electrons at emission is $k_{B} T=0.1 \mathrm{eV}$. The normalized transverse rms emittance at the cathode surface (radius $R$ ) is[5]:

$$
\tilde{\varepsilon}_{n, t h}=\frac{R}{2}\left(\frac{k_{B} T}{m c^{2}}\right)^{1 / 2}
$$

in our case $\tilde{\varepsilon}_{n, t h}=0.33 \mathrm{~mm}$ mrad.

The control grid in front of the cathode also has a defocusing effect on the beam. In order to reduce the calculation cost, this effect was calculated in a separate simulation in TS3, whereas the complete gun was calculated in TS2, exploiting the rotational symmetry.

Although the TS3 calculation includes space charge effects, it is in good agreement with an analytical expression for the normalized rms emittance acquired by a beam after passing the wire mesh, which is given by Reiser[5]:

$$
\tilde{\varepsilon}_{n, g}=\frac{R a}{4}\left(\frac{2 e V_{g}}{m c^{2}}\right)^{1 / 2} \frac{\left|E_{2}-E_{1}\right|}{4 V_{g}}
$$

where $2 a$ is the distance between mesh wires, $V_{g}$ the grid voltage, and $E_{1}$ and $E_{2}$ the electric field on either side of the mesh. In our case $\tilde{\varepsilon}_{n, g}=0.35 \mathrm{~mm} \mathrm{mrad}$.

If the intrinsic thermal emittance is included, one obtains for the normalized rms emittance at the cathode grid the result

$$
\tilde{\varepsilon}_{n}=\left(\tilde{\varepsilon}_{n, t h}^{2}+\tilde{\varepsilon}_{n, g}^{2}\right)^{1 / 2} .
$$

\subsection{Simulation with MAFIA-TS2}

The TS2 simulation may use the result of the TS3 calculation as initial condition. However, in order to reduce the number of macro particles in the calculation, the beam was initialized with zero emittance. Nevertheless, we used more than 70000 macro particles and the mesh step for the
FDTD grid was $250 \mu \mathrm{m}$. Since this is larger than the distance of the control grid from the cathode, the initial energy of the electrons was set to $10 \mathrm{eV}$, which corresponds to the grid voltage at the center of the bunch. Fig. 2 shows the results of this calculation.
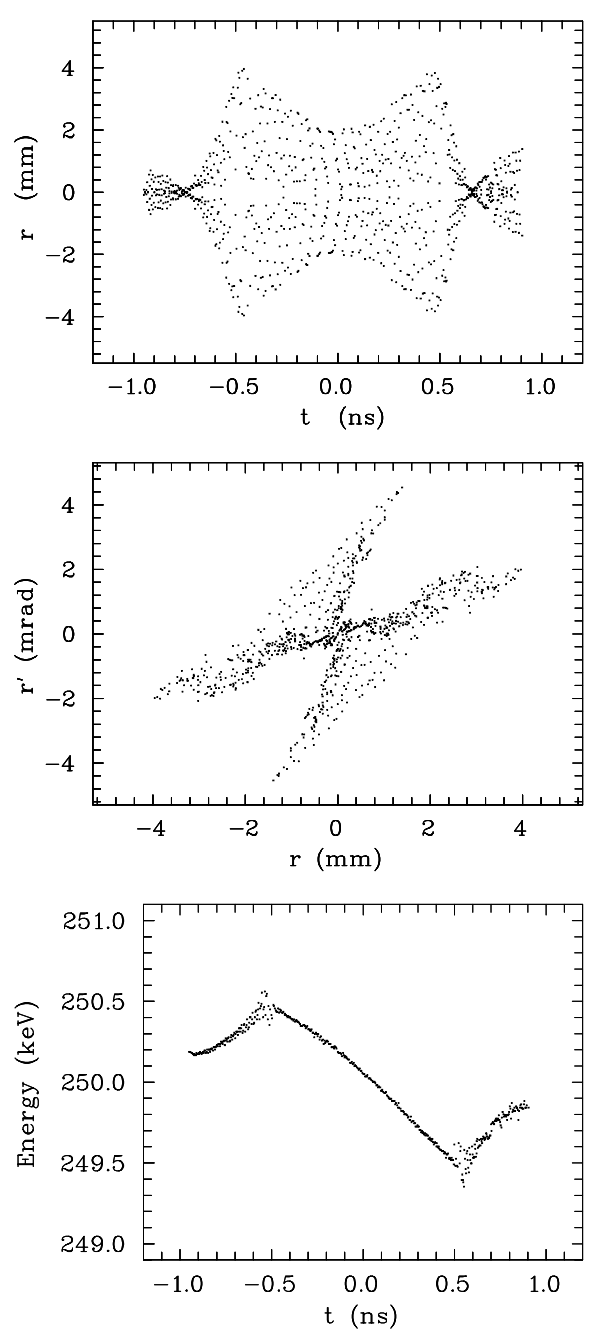

Figure 2: Distributions as calculated by MAFIA-TS2 at the exit of the gun. From top to bottom the figures show the radius as a function of time, the transverse phase space and the longitudinal phase space.

All plots show the influence of the space charge. Especially, a correlated energy spread is acquired by the bunched beam: particles in the head of the bunch are accelerated by space charge forces, while particles in the tail are decelerated. This effect is much larger than the uncorrelated energy spread. Another space charge effect can be observed in the transverse plane: particles in the head and tail of the bunch are focused more strongly than those in the center. This increases the projected emittance; however, those particles will be discarded at the chopper and therefore do not contribute to the beam quality in the FEL. The numerical results are summarized in table 1 . When the initial emittance is included, the transverse emittance increases to $0.86 \mathrm{~mm}$ mrad. Since the axisymmetric cal- 
culation in TS2 excludes any misalignment effects, these values give a lower limit for the actual emittance.

\begin{tabular}{|l|r|}
\hline normalized transverse rms emittance & $0.7 \mathrm{~mm} \mathrm{mrad}$ \\
normalized longitudinal rms emittance & $51.3 \mathrm{eV} \mathrm{ns}$ \\
beam radius $\sigma_{x}$ & $1.5 \mathrm{~mm}$ \\
beam divergence $\sigma_{x^{\prime}}$ & $0.8 \mathrm{mrad}$ \\
pulse length $\sigma_{t}$ & $332 \mathrm{ps}$ \\
energy spread $\sigma_{E}$ & $155 \mathrm{eV}$ \\
\hline
\end{tabular}

Table 1: Beam parameters at the end of the electrostatic cascade according to the simulation with MAFIA-TS2.

\subsection{Simulation with GPT}

The calculation with GPT was started with the parameters given above for the thermionic cathode. The influence of the control grid was neglected. 1000 particles were used.

Table 2 shows the numerical results. GPT yields the same space charge effects discussed above with a tendency to higher uncorrelated momentum and energy spread. An additional calculation starting with $10 \mathrm{eV}$ particle energy and zero emittance yielded similar results, confirming that this is not a physical effect in the low energy part, which was neglected in TS2.

\begin{tabular}{|l|r|}
\hline normalized transverse rms emittance & $1.55 \mathrm{~mm} \mathrm{mrad}$ \\
normalized longitudinal rms emittance & $248 \mathrm{eV} \mathrm{ns}$ \\
beam radius $\sigma_{x}$ & $1.1 \mathrm{~mm}$ \\
beam divergence $\sigma_{x^{\prime}}$ & $1.4 \mathrm{mrad}$ \\
pulse length $\sigma_{t}$ & $294 \mathrm{ps}$ \\
energy spread $\sigma_{E}$ & $228 \mathrm{eV}$ \\
\hline
\end{tabular}

Table 2: Results of the simulation with GPT.

\section{MEASUREMENT OF BEAM PARAMETERS}

In order to check the predictions of the simulations and to verify the validity of the assumptions made therein, beam parameters were measured in the front section of the injector beamline of the S-DALINAC.

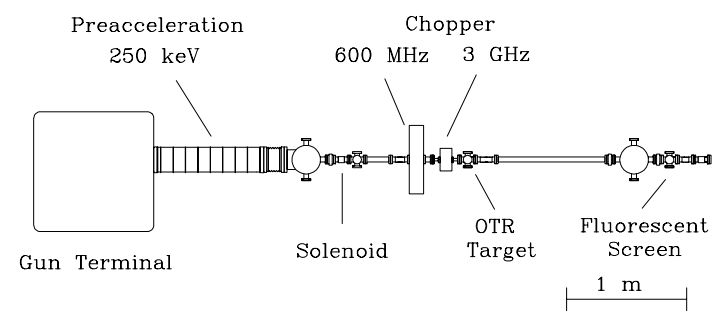

Figure 3: Setup for the measurement of the beam parameters.

The transverse parameters were determined a short distance downstream of the electrostatic acceleration tube (see fig. 3). The two-dimensional intensity distribution of the beam on a thin aluminum foil was measured as a function of the focal strength of the solenoidal lens located upstream, by detecting and recording the visible part of the emitted transition radiation (OTR) by a standard CCD camera, framegrabber board, and a PC. Fitting a parabola to the measured beam sizes (see fig. 4) yields the complete set of transverse beam parameters at the location of the lens. The resulting figures for normalized emittance, beam radius, divergence, and correlation are given in the inset in fig. 4 .

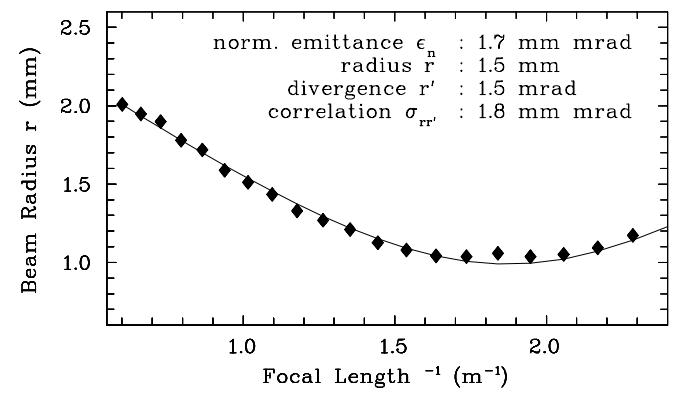

Figure 4: Beam size as a function of the inverse focal length of the solenoid lens. From the fitted curve, the transverse beam parameters were obtained.

In the front end of the injector beamline of the SDALINAC diagnostics for the energy spread of the $250 \mathrm{keV}$ beam is not available. The pulse length however could be determined by deflecting the beam vertically by a $600 \mathrm{MHz}$ and horizontally by a $3 \mathrm{GHz}$ chopper cavity. From the resulting Lissajous figures observed on a fluorescent screen further downstream (right edge of fig. 3) a pulse duration of 600-700 ps (FWHM) was deduced.

\section{CONCLUSION}

We used two different programs to simulate beam dynamics in the S-DALINAC electron gun. Both yield similar results on space charge effects. The measured values for the transverse phase space and the pulse length are in remarkably good agreement with the simulation results. For the first time, longitudinal phase space was considered in the gun of the S-DALINAC.

We are therefore convinced that the results obtained here can be used successfully in simulations of the fairly complex beam dynamics in the remaining sections of the $S$ DALINAC injector.

\section{REFERENCES}

[1] K.Alrutz-Ziemssen et.al., Nucl. Instr. Meth. A304 (1991), p.300.

[2] The MAFIA collaboration, User's Guide MAFIA Version 4.00, CST GmbH, Lauteschlägerstr. 8, D-64289 Darmstadt, Germany.

[3] U.Becker, M.Dohlus, T.Weiland, Three Dimensional Klystron Simulation, Particle Accelerators, 1995, Vol. 51, pp. 135-154.

[4] GPT User Manual, Pulsar Physics, Flamingostraat 24, 3582 SX Utrecht, The Netherlands.

[5] M.Reiser, Theory and Design of Charged Particle Beams, John Wiley \& Sons, New York, 1994. 